\title{
Premier succès d'Alara à Golfech
}

\author{
P. PUJOL*
}

RÉSUMÉ

\begin{abstract}
Electricité de France et son parc de centrales nucléaires se sont engagés depuis 1991 dans une politique d'optimisation de la radioprotection. Au centre de production nucléaire de Golfech, un des 20 sites nucléaires français, une démarche ALARA a été entreprise immédiatement et le résultat obtenu lors de la première visite complète de sa première unité de production, visite qui représente plus de 400000 heures de travail, est très encourageant. Cet article explique comment, sous l'impulsion de la direction et du comité ALARA, la démarche concourt, par une mobilisation et par une motivation de tous les acteurs, à des résultats excellents.
\end{abstract}

ABSTRACT First ALARA success at Golfech.

Since 1991, Electricité de France nuclear power plants have started the implementation of a policy of radiation protection optimisation. At the Golfech nuclear power station, one of the 20 french nuclear sites, an ALARA procedure was immediately set up and the results obtained during unit 1 first outage (an inspection representing over 400000 hours'work) were most encouraging. The paper explains how - under the impetus of the management and ALARA committee - the procedure worked towards excellent results thanks to every actors'mobilization and motivation.

\section{Introduction}

Electricité de France dispose d'un parc nucléaire conséquent (plus de 50 unités). Pour les plus anciennes unités de $900 \mathrm{MW}$, se pose le problème des grands chantiers. Le remplacement de générateurs de vapeur réalisé à Dampierre, qui est l'exemple type, a été l'occasion de la mise en place d'une démarche "ALARA" qui a été décrite dans cette revue [2].

Les centrales les plus récentes de $1300 \mathrm{MW}$ bénéficient d'améliorations de conception qui visent à limiter les sources de rayonnement. Malgré cela, on observe une lente dégradation des résultats en terme d'exposition collective. La direction du parc nucléaire de France en créant un comité ALARA PARC, a donc voulu que les exploitants recherchent une amélioration de l'exposition collective pour leurs opérations de maintenance.

\footnotetext{
* Centre nucléaire de production d'électricité de Golfech, Boîte Postale 24, 82400 Golfech.
} 
Afin de se préparer aux nouvelles contraintes réglementaires et comme les arrêts de tranche occasionnent la prise de plus de $80 \%$ de la dose annuelle (agents d'Electricité de France et agents d'entreprises prestataires), le direction du centre de Golfech a décidé d'appliquer une structure ALARA dès le premier arrêt de la tranche 1 (1300 MW) pour sa première visite complète de manière à ne pas laisser prendre de mauvaises habitudes sur le site. Pour rendre effective dans les faits cette volonté, un effort permanent de mobilisation des acteurs, la mise en œuvre de procédures et d'actions spécifiques, ont permis de réussir un arrêt de tranche de 103 jours avec une exposition collective inférieure à $1 \mathrm{H} \cdot \mathrm{Sv}$ et une exposition individuelle inférieure à $7 \mathrm{mSv}$ pour plus de $98 \%$ des intervenants ; ce résultat est le meilleur réalisé dans une centrale nucléaire française.

\section{Mise en place de structures spécifiques pour l'arrêt}

Une visite complète nécessite la mise en place d'une structure spécifique. A Golfech, cette structure a été voulue légère mais forte et soudée, de manière à s'appuyer sur la structure perenne, celle des services opérationnels. Pour maîtriser l'exposition collective, un comité a été créé et la structure permanente des agents de radioprotection a été renforcée.

\subsection{Création d'un comité ALARA}

Cinq mois avant le début de l'arrêt, la direction du site de Golfech a créé un comité regroupant tous les services opérationnels et le chef de l'arrêt. Ce comité, présidé par le chef de centrale délégué, était piloté par le chef de la section "Sécurité-Radioprotection" selon une lettre de mission lui donnant délégation pour pouvoir, après analyse, prendre toute décision d'organisation. La présence dans le comité des agents responsables dans leur service de la bonne réussite de l'arrêt, et tout particulièrement celle du chef d'arrêt, est à notre avis une clé de notre réussite. C'est ainsi que le comité ALARA a permis de définir en particulier:

- un objectif dosimétrique clair et volontariste,

- un examen des chantiers à exposition forte.

Dans un souci d'équité entre tous les intervenants (agents EDF ou agents d'entreprise), les objectifs dosimétriques individuel et collectif retenus pour l'arrêt de tranche par le comité étaient :

- objectif de dose individuelle pour l'arrêt de tranche :

- dose $<7 \mathrm{mSv}$ pour plus de $95 \%$ de tous les intervenants,

- dose $<20 \mathrm{mSv}$ pour tout le monde;

- objectif d'exposition collective : d'abord évalué à 1,6 H.Sv et recalé en cours d'arrêt à $1 \mathrm{H} \cdot \mathrm{Sv}$ (grâce, en particulier, à l'obtention d'un bon indice d'activité du circuit primaire). 
Afin d'améliorer les expositions, des efforts ont été entrepris plus particulièrement sur les chantiers suivants : robinetterie, servitudes, calorifuge, ouverture/fermeture cuve, échafaudages.

\subsection{Renfort de l'équipe de radioprotection}

La section "Sécurité-Radioprotection" a dû être renforcée par une entreprise extérieure qui travaillait sous son autorité en binôme : 4 agents du centre, 4 agents d'entreprises dont 1 affecté au suivi quotidien des résultats et 3 affectés à la permanence de suivi des chantiers en zone contrôlée. Les agents ont travaillé en horaire normal sauf pour les travaux en génératrice inférieure où ils étaient en $2 \times 8$.

\section{Motivation de tous les acteurs}

Pour l'atteinte des objectifs du comité ALARA du centre de Golfech, nous avions misé sur une implication de tous les acteurs que sont les agents du centre et les agents d'entreprises, le tout dans un esprit de partenariat.

\subsection{Avant le chantier de la première visite complète}

A l'initiative du chef d'arrêt, et avec une participation effective de la direction du centre de Golfech, tous les chefs d'entreprises extérieures ont été invités 2 semaines avant le début du chantier, dans le cadre d'une journée de préparation et d'information des prestataires. Nous avons ainsi expliqué, en particulier, le concept ALARA et exposé les actions qui allaient être engagées [1]. Les chefs de chantier des entreprises intervenant lors de la visite complète, acteurs véritablement importants puisqu'en prise directe sur les intervenants des entreprises, ont été motivés lors des réunions d'enclenchement précédant le début des travaux. Notre intervention a permis de rappeler le concept ALARA et ses applications probables sur le chantier et surtout d'échanger nos idées sur ce sujet.

Enfin, l'ensemble des intervenants prestataires a été informé des objectifs ALARA par une sensibilisation individuelle au cours d'un entretien postérieur au test de radioprotection réalisé le jour de leur arrivée sur le site. De plus, lors de cet accueil sur le site, les intervenants ont reçu une plaquette d'information ALARA.

Dans le cadre de la préparation à cet arrêt de la tranche 1, la section "Sécurité-Radioprotection" a effectué des interventions de 1 à $2 \mathrm{~h}$ au cours de formation effectuée dans chaque service de l'usine. C'est ainsi qu'on leur a présenté :

- le concept ALARA et l'application pratique à Golfech,

- l'application sur leurs chantiers principaux,

- les fiches ALARA destinées aux agents chargés d'opérations. 
Cela a permis un échange de vue, une discussion avec les intervenants. La hiérarchie des services opérationnels a apporté son soutien à cette action.

Ainsi donc, avant le début de l'arrêt, tout acteur, qu'il appartienne à EDF ou à des entreprises extérieures, connaissait ALARA, le pourquoi et le comment de cette politique et son enjeu pour le site.

\subsection{Pendant le chantier}

Le dialogue a été permanent entre les opérationnels sur le terrain. En premier lieu, la cellule des agents responsables de l'arrêt a travaillé en intégrant le facteur dosimétrique dans les décisions opérationnelles et avant de lancer certaines opérations importantes. Ensuite, la présence des agents de radioprotection (centrale et entreprises) sur le terrain a permis de renforcer l'information des intervenants et de leur apporter le soutien nécessaire au bon déroulement des chantiers. Ainsi, on a pu leur expliquer "la chasse aux entrées inutiles" en zone contrôlée et "la chasse à la production abusive de déchets" (vinyle, chiffons...). Les partenaires sociaux, au sein du CSHSCT (Comité spécial d'hygiène et de sécurité et des conditions de travail) ont été, eux aussi, informés des objectifs et des résultats de la démarche ALARA lors de quatre réunions (une avant le début de l'arrêt, trois pendant la durée des travaux). Ces réunions ont permis de faire progresser la démarche car, grâce à une écoute mutuelle, un véritable climat de confiance s'est instauré et certaines entreprises ont eu des apports intéressants, telles que : Wanner Isofi pour le calorifuge, Polinorsud pour les servitudes et Framatome pour les contrôles sur la cuve du réacteur.

En même temps que la mobilisation était présente sur le terrain, nous avons réalisé un gros effort de communication interne des résultats en matière dosimétrique. C'est ainsi qu'une campagne d'affichage a permis de résumer l'action ALARA sur douze points stratégiques du site grâce à des affiches changées régulièrement. Cette permanence visuelle a été complétée par un encart systématique de l'évolution des résultats dosimétriques dans le journal hebdomadaire de l'arrêt distribué à tous les agents intervenants (agents EDF et entreprises). Ce journal a été le catalyseur de cet arrêt et a permis d'associer tous les acteurs aux résultats et au succès final. Enfin, des tracts affichés partout et ciblés sur les deux thèmes suivants : garder le cap ALARA et performance inférieure à $1 \mathrm{H} . \mathrm{Sv}$, ont permis aux acteurs de ne pas oublier cet enjeu.

\section{Procédures et actions mises en cuvre}

Pour atteindre l'objectif, nous avons agi sur le terme source, sur les écrans et sur les temps d'exposition. 


\subsection{Actions sur le terme source}

\section{a) Obtention d'une épuration poussée du circuit primaire principal}

Avant de rompre l'intégrité du circuit primaire qui contient le terme source hormis celui constitué par les assemblages de combustible, il faut oxygéner le circuit et l'épurer de manière à atteindre des valeurs en iode 131 et en xénon 133 inférieures à des seuils fixés. Nous avons décidé de prendre $24 \mathrm{~h}$ en plus du planning initial, pour atteindre des valeurs plus restrictives que celles recommandées dans les spécifications chimiques. Nous avons ainsi gagné un facteur 7 sur l'activité du circuit primaire en iode 131 et un facteur 14 pour le xénon 133. Cela nous a permis d'avoir un circuit primaire, représenté par son indice d'activité, peu contaminé $(0,26 \mathrm{mSv} / \mathrm{h})$ et une activité du produit d'activation le plus important qu'est le cobalt 58 de $550 \mathrm{mBq} \mathrm{m}^{3}$ au lieu de $1000 \mathrm{mBq} \mathrm{m}^{3}$ correspondant à la spécification. Cette opération, coûteuse en temps, mais à coup sûr bénéfique pour la réduction des expositions a, semble-t-il, permis de baisser d'environ $20 \%$ le débit de dose lié à l'activité de l'eau du circuit primaire principal.

\section{b) Modifications des conditions d'intervention}

La présence sur le terrain de représentants de la section "SectionRadioprotection" et l'implication totale des acteurs a permis de modifier ponctuellement certaines conditions d'intervention en jouant sur les protections tant à la source que dans l'environnement.

Quatre exemples:

- remplissage des tuyauteries environnantes du circuit primaire principal pour les intervenants sur les trous d'œil et trous de poing des générateurs de vapeur;

- travail sous eau pour le contrôle par ultrasons de la plaque tubulaire des générateurs de vapeur;

- pose de protections biologiques sur les piquages du pressuriseur dès le décalorifugeage ;

- décontamination d'organes irradiants (vannes, clapets) avant leur démontage et réparation à l'atelier.

\subsection{Action sur l'élaboration du planning}

Le chef d'arrêt, membre permanent du comité ALARA de la centrale, a intégré la démarche ALARA dans l'élaboration du planning de l'arrêt. C'est ainsi qu'il a placé l'épreuve hydraulique en début d'arrêt de manière à reporter à plus tard les travaux d'entretien notamment sur la robinetterie en génératrice inférieure et les interventions de contrôle sur les générateurs de vapeur pour laisser décroître l'activité des circuits.

\subsection{Action sur le temps de travail}

Une limitation drastique du nombre d'accès en zone contrôlée pour les visiteurs a permis de n'y faire rentrer que les intervenants. C'est ainsi 
que les agents de l'usine qui n'avaient pas de travail en zone contrôlée ont vu leur accès interdit. Nous avons dû faire exception pour les autorités locales et les journalistes, mais en veillant qu'ils intègrent dans leur circuit de visite le moins de dose possible. De cette façon, nous n'avons eu que 1004 intervenants en zone contrôlée (le nombre moyen habituel varie de 1000 à 1500 selon les sites et les activités).

La présence assidue d'agents sensibilisés par la dosimétrie a permis de réduire les inobservations des règles de radioprotection par les intervenants et les a incités à quitter les lieux les plus "chauds" dès la fin de leur intervention.

\section{Résultats}

La visite complète du réacteur $n^{\circ} 1$ du centre de Golfech a été un succès du point de vue dosimétrique puisque nous avons eu une exposition collective de $981 \mathrm{H} \cdot \mathrm{mSV}$ alors que le meilleur résultat, à cette époque sur les tranches $1300 \mathrm{MW}$, était de $1390 \mathrm{H} \cdot \mathrm{mSv}$ pour l'arrêt de Cattenom 2. L'objectif qui était de $1 \mathrm{H} \cdot \mathrm{Sv}$ n'a donc pas été dépassé. Ce résultat a été obtenu avec 1000 intervenants en zone contrôlée répartis en 2/3 d'agents d'entreprises et 1/3 d'agents EDF (Tab. I).

TABLEAU I

Bilan dosimétrique

Dosimetric statistics

\begin{tabular}{|lc|}
\hline Dose collective ( $\mathrm{H} \cdot \mathrm{msv})$ & 980,95 \\
\hline Nombre d'intervenants en zone contrôlée & 1004 \\
\hline Dose moyenne par intervenant (msv) & 0,98 \\
\hline
\end{tabular}

L'évolution de la dosimétrie collective est donnée en figure 1. Au niveau des expositions individuelles (fig. 2), les objectifs ALARA ont été, eux aussi, atteints puisqu'aucun agent n'a eu une exposition excédant $20 \mathrm{mSv}$, et que $98 \%$ des agents ont eu une dose inférieure à $7 \mathrm{mSv}$, pour un objectif de $95 \%$. La répartition de la dose individuelle montre que les agents d'entreprise sont les plus exposés : 19 agents ayant reçu une dose supérieure à $7 \mathrm{mSv}$ dont 12 une dose supérieure à $10 \mathrm{mSv}$.

On retrouve, parmi les entreprises les plus touchées, celles qui procèdent aux opérations de décalorifugeage et calorifugeage des circuits et matériels et celles qui assurent les servitudes (nettoyage, réalisation d'échafaudage). L'examen des deux catégories de prestations est un axe d'amélioration pour les années futures. II convient de préciser qu'à la réussite en terme de dosimétrie collective de cet arrêt de tranche on peut rajouter les résultats satisfaisants en matière de respect du planning et d'effluents solides. 


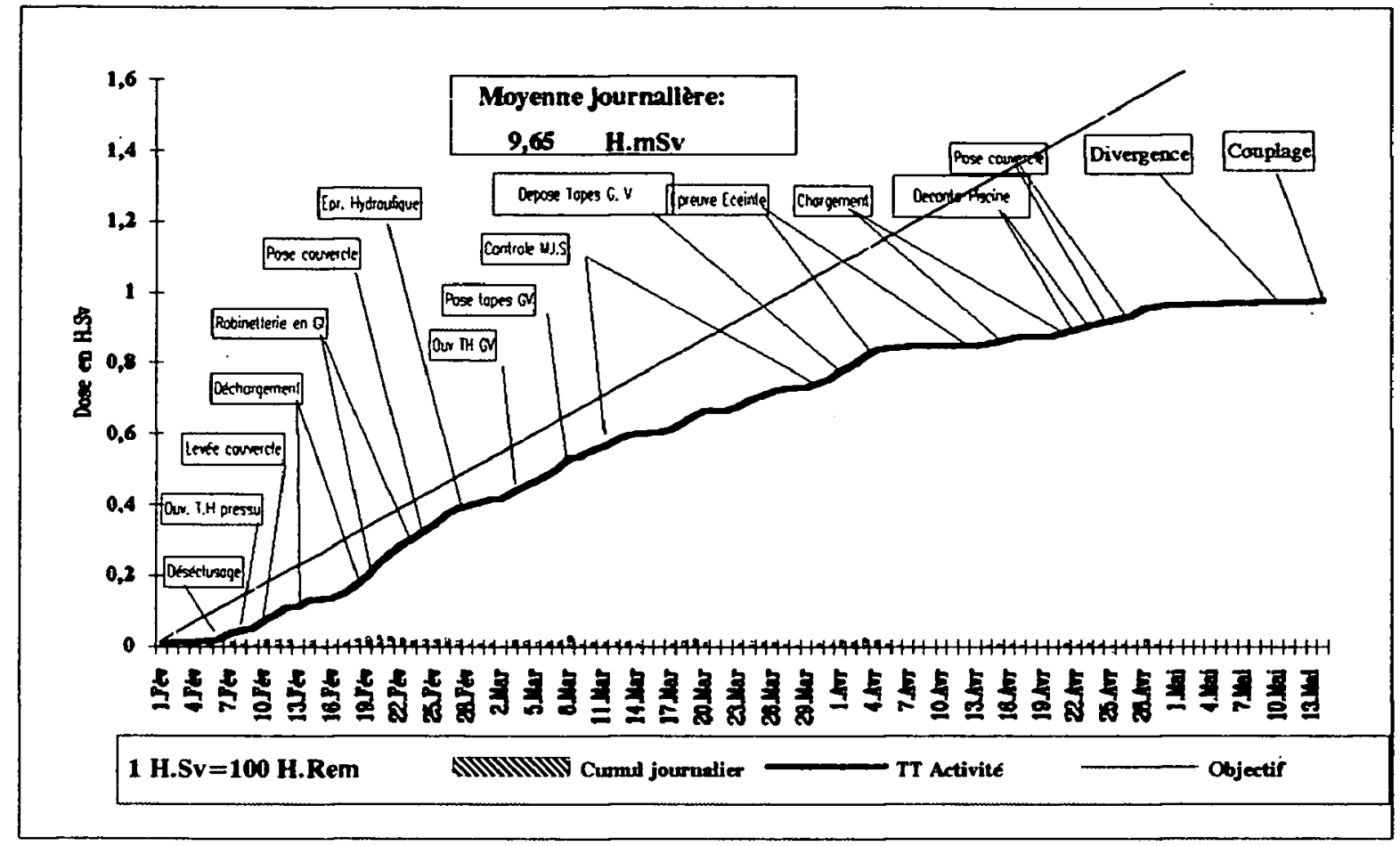

Fig. 1. - Evolution journalière des doses collectives. Daily trends of collective doses

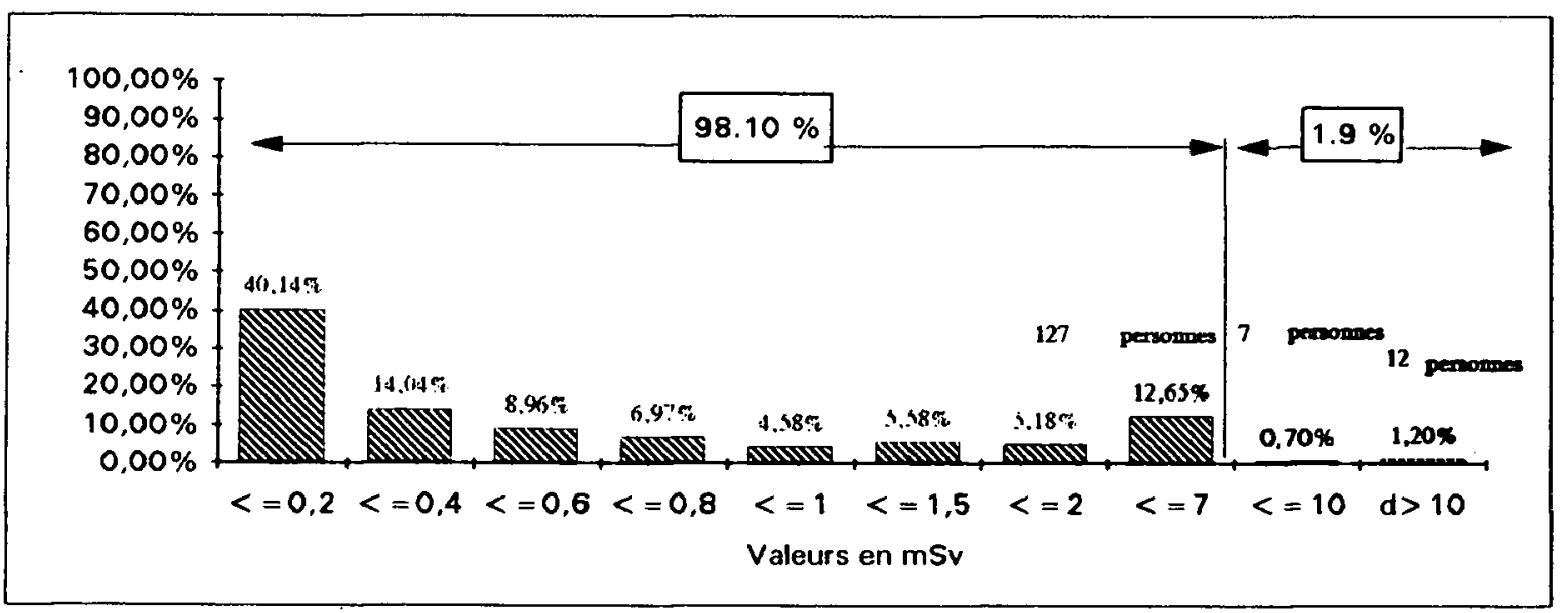

Fig. 2. - Répartition de la dose individuelle. Distribution of Individual doses

Les objectifs tant en matière d'exposition collective (objectif : $1 \mathrm{H} \cdot \mathrm{Sv}$, réalisé : $0,98 \mathrm{H} \cdot \mathrm{Sv}$ ) que de répartition individuelle ont été atteints :

- objectif : $95 \%$ des intervenants avec une dose inférieure à $7 \mathrm{mSv}$ et $100 \%$ avec une dose inférieure à $20 \mathrm{mSv}$;

- réalisation : $98 \%$ des intervenants avec une dose inférieure à $7 \mathrm{mSv}$ et $100 \%$ avec une dose inférieure à $20 \mathrm{mSv}$. 


\section{Conclusion}

L'objectif dosimétrique visé pour cette première expérience avec la démarche ALARA a été atteint car ce challenge a été adopté par tout le personnel intervenant sur l'arrêt de tranche au même titre que les deux autres challenges suivants, eux aussi atteints, à savoir le respect de la durée de l'arrêt et l'obtention du minimum de déchets solides possibles. Selon nous ces trois démarches vont de pair et doivent être menées en même temps.

On ne peut espérer $y$ arriver que si la confiance règne entre tous les nombreux partenaires. C'est pourquoi il faut une mobilisation générale voulue par la hiérarchie pour donner à la démarche ALARA une place importante dans l'arrêt de tranche. Le bon résultat collectif de cet arrêt doit nous servir de référence sur le site pour espérer être demain encore plus performant, ce qui est possible en améliorant, en particulier, la préparation et la planification des interventions avec le concept ALARA.

P.S. : Le CNPE de Golfech vient de terminer, en 1983, sur la tranche 1, sa première visite partielle avec un excellent résultat dosimétrique: $0,448 \mathrm{H} \cdot \mathrm{Sv}$. Ce résultat confirme le succès précédent et représente la meilleure performance jamais atteinte sur un site du parc nucléaire français [3].

\section{REFERENCES}

[1] INTERNATIONAL COMMISSION ON RADIOLOGICAL PROTECTION (ICRP) Recommendations... (ICRP publication 60). Oxford : Pergamon press, 1991.

[2] JURION C., BLAIN A., LEFAURE C. - Gestion ALARA de la protection radiologique lors des remplacements de générateurs de vapeur de Dampierre 1 (France) en 1990. Radioprotection, 1992, 27(2) 157-171.

[3] STRICKER L., DOLLO R., PERIN M. - ALARA programme management and organisation in EDF nuclear power stations. In : CEC 4th European seminar on Optimisation of radiation protection, Luxembourg, April 20-22, 1993 (à paraître). 